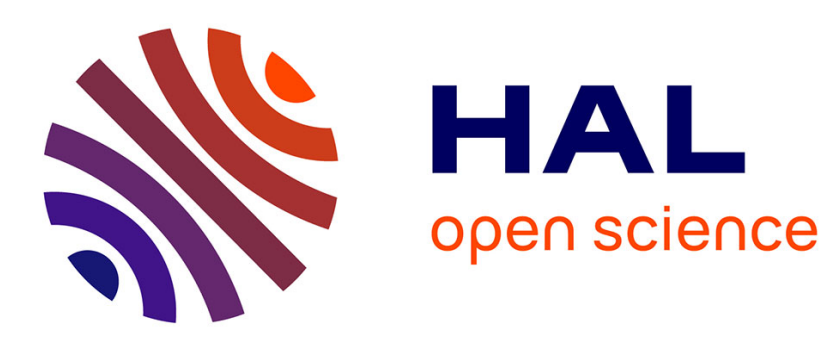

\title{
Periodic structures in colloidal crystals with oscillatory flow
}

\author{
W.D. Dozier, P.M. Chaikin
}

\section{To cite this version:}

W.D. Dozier, P.M. Chaikin. Periodic structures in colloidal crystals with oscillatory flow. Journal de Physique, 1982, 43 (6), pp.843-851. 10.1051/jphys:01982004306084300 . jpa-00209461

\section{HAL Id: jpa-00209461 https://hal.science/jpa-00209461}

Submitted on 1 Jan 1982

HAL is a multi-disciplinary open access archive for the deposit and dissemination of scientific research documents, whether they are published or not. The documents may come from teaching and research institutions in France or abroad, or from public or private research centers.
L'archive ouverte pluridisciplinaire HAL, est destinée au dépôt et à la diffusion de documents scientifiques de niveau recherche, publiés ou non, émanant des établissements d'enseignement et de recherche français ou étrangers, des laboratoires publics ou privés. 
Classification

Physics Abstracts

$47.60-47.15-82.70 \mathrm{D}$

\title{
Periodic structures in colloidal crystals with oscillatory flow (*)
}

\author{
W. D. Dozier and P. M. Chaikin \\ Department of Physics, University of California, Los Angeles, California 90024, U.S.A.
}

(Reçu le 23 décembre 1981, accepté le 25 février 1982)

\begin{abstract}
Résumé. - Nous avons étudié quelques aspects de l'écoulement de cristaux colloidaux dans un capillaire. En appliquant une pression oscillante nous induisons un écoulement dans la région de cisaillement proche où le cristal a une réponse plastique. Dans certaines conditions nous observons des régions cristallines bien orientées avec un dessin d'orientation périodique le long du tube. Puisque les cristaux ont une périodicité d'environ $0,5 \mu$, visible en diffraction de lumière, on observe un dessin coloré. Nous avons étudié la formation de ces striures en fonction du module de cisaillement, de la fréquence, de l'amplitude des oscillations, de la taille des particules et du diamètre du tube.
\end{abstract}

\begin{abstract}
We have studied some aspects of the flow of colloidal crystals in a capillary. Applying oscillatory pressure we induce flow in the shear region near where the crystal responds plastically. Under certain conditions, we observe well oriented crystalline regions which alternate orientations in a periodic pattern along the tube. Since the crystals have a lattice spacing of approximately $0.5 \mu$ and Bragg scatter visible light, a periodic colour pattern is observed. We have studied the formation of these cylindrically symmetric striations as a function of shear modulus, frequency and amplitude of oscillations, particle size and tube diameter.
\end{abstract}

1. Introduction. - Monodisperse charged colloids have been of considerable interest in recent years as a model system for a number of problems dealing with colloid physics and with liquid-solid transitions [1-8]. Under certain conditions of colloid charge, particle density and concentration of electrolyte in the solvent the repulsive screened Coulomb interaction is sufficiently strong to cause the suspension to crystallize into a "classical Wigner solid ». For particle densities of the order of $10^{13}$ particles $\mathrm{cm}^{-3}$ the characteristic unit cell size is approximately $0.3 \mu$ and the crystals Bragg scatter visible light. This gives the colloidal crystals an opalescence which easily distinguishes the ordered crystalline state from the liquid state.

For a charge per sphere of approximately 1000 electronic charges and a separation of about $0.3 \mu$ the electrostatic interaction between a pair of charged spheres is comparable to the interaction between atoms in a conventional solid. However, since the particle density is nearly 10 orders of magnitude less than that in a conventional solid, the elastic constants are proportionately smaller $\left(\approx 10^{12} \mathrm{dyn} . \mathrm{cm}^{-2}\right.$ in

$\left(^{*}\right)$ Research supported by ONR under contract \# N00014-76-C-1078 and A. P. Sloan Foundation (PMC). metals, $\approx 100 \mathrm{dyn} . \mathrm{cm}^{-2}$ in colloidal crystals) [9]. This allows for the study of a hydrodynamic regime which is usually inaccessible in conventional solids or liquids. The ratio of shear rate to compressibility or shear modulus may be taken to much higher values in colloidal crystals. Recently this property has been used to investigate shear melting and anisotropic compression in colloids [10].

In this paper, we are interested in the intermediate flow regime where the crystalline phase persists although the shear rate is relatively large. For flow in a capillary, we distinguish three regimes. At low oscillatory shear rate, the colloidal crystal responds elastically. This is easily observed by an oscillatory colour change of the Bragg scattered light indicating a shear strain. At higher shear rates, the crystal responds plastically and extremely inhomogeneously. Crystallites change their boundary configurations and reorient. This is the regime covered in the present work. Finally at higher shear rates the crystal is destroyed and no three dimensional Bragg scattering is observed. The sample then responds like a fluid.

The fact that the shear modulus and hence the yield stress are quite small $[5,11,12]$ is what makes the hydrodynamics highly nonlinear and thus interesting. The instability toward the formation of periodic 
domains that we have seen is only one of many which must be intrinsic to such systems.

2. Experiments. - The samples used in this study were prepared from monodisperse polystyrene spheres commercially available from Dow Diagnostics. The colloid latex was diluted to the desired concentration by adding deionized water. Ion exchange resin was also added [13]. After several days of setting, the samples crystallize, as can be seen by the appearance of characteristic opalescence. For these experiments, we used $0.109 \mu$ and $0.220 \mu$ diameter spheres in the volume fraction regime from 0.100 to 0.005 (particle density $\approx 10^{13} \mathrm{~cm}^{-3}$ ). The samples were characterized by electron micrography, conductimetric titration and most germane to this work, elastic shear modulus measurements [12].

The capillary tubes ranged in inner diameter from 1 to 4 millimeters. They were cleaned by a flow of deionized water for small diameters, or allowing them to stand in water with ion exchange resin for the larger tubes. Having used many different glasses in the capillary tubes, and different cleaning treatments, our results do not seem to depend on the preparation of the glass surface as long as they are deionized sufficiently to prevent melting of the crystal.

For the smaller diameter tubes, the capillary was lowered into a crystallized latex which then rose $\approx 1 \mathrm{~cm}$ up the capillary from surface tension forces. This geometry is shown in the photo in figure $1 a$. A plastic tube which was connected to an oscillatory pressure source (a speaker with a sealed front) was then attached to the top of the capillary so that a driven oscillatory flow could be effected.

The photo (Fig. 1) was taken with a light source at about $45^{\circ}$ directly above the camera lens. The bright stripes to either side of the centre of the tube are orange in colour and result from Bragg scattering in the highly polycrystalline sample (in this case, $0.109 \mu$ diameter spheres, $1.47 \times 10^{13} \mathrm{~cm}^{-3}$ ). The fact that we observe stripes rather than random "flecks" of orange indicates that one plane of the crystallites has a preferred direction parallel to the surface of the tube while the other directions are random.

When small amplitude oscillations were produced, the orange lines periodically changed colour from red

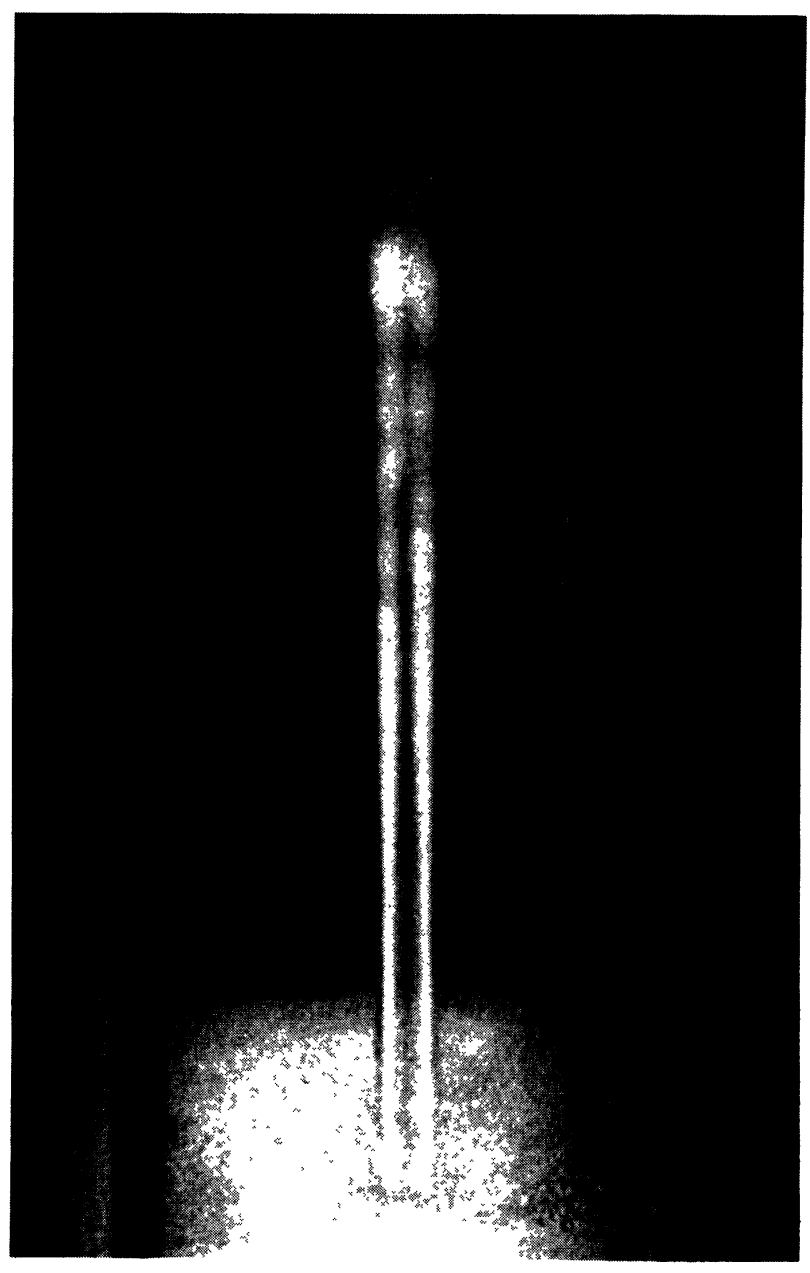

a)

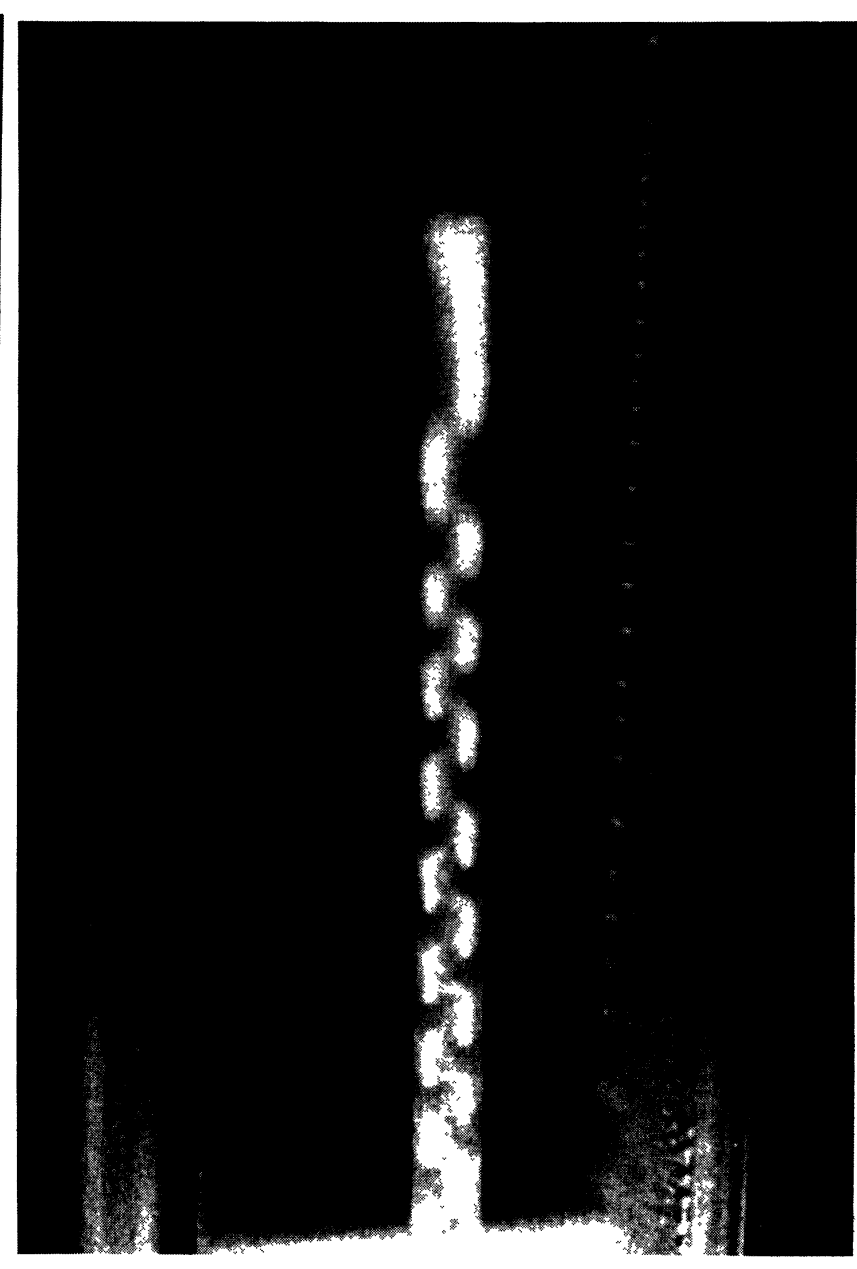

b)

Fig. 1. - a) Colloidal crystal in a capillary before being driven. Bright stripes are due to Bragg scattering. $b$ ) Same configuration after driven for several minutes at $10 \mathrm{~Hz}$. 
shifted to green shifted. This indicates elastic deformation of the crystallites. The Bragg scattering planes are spaced closer or further apart than in the absence of the shear strain field.

For larger amplitude flow, there is a time $(1 \mathrm{~min} .-1 \mathrm{~h})$ after the initial increase during which the appearance of the sample changes until finally a steady state is established. Regions which are red and green to the eye appear in a "checkerboard" pattern along the length of the tube. This is shown in figure $1 b$, unfortunately in black and white. The body of this paper is concerned with describing this periodic pattern.

When using larger diameter tubes, the capillary action is not sufficient to produce a long enough column of latex to observe in the geometry described above. We therefore made several " $U$ » tubes in which one end was left open while the other end was attached to the oscillating pressure as before. As for the previous geometry, when oscillations of sufficient amplitude were produced, a spatially modulated colour pattern was observed.

While the physical situation is not apparent from the pictures in figure 1 or from casual inspection of the driven latex, a more careful visual analysis with the aid of a microscope shows that the phenomenon may be described by the schematic shown in figure 2 . With a

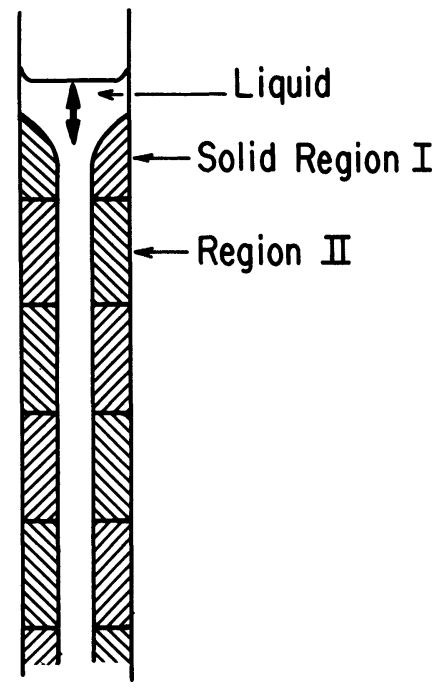

Fig. 2. - Schematic showing axially symmetric, mirror image, alternating pattern of solid regions with a fluid core.

specific configuration of light source, sample and observer, an angle is defined such that only certain Bragg scatterings are allowed, corresponding to particular colours' and a particular orientation and spacing of crystal planes. For the particle densities we are using, the scattering results from the first $0.1 \mathrm{~mm}$ and we are thus observing a surface effect. A particular band (region I) consists of crystallites which are well oriented with respect to the direction of flow and the perpendicular to the surface of the tube. The orientation is therefore axially symmetric and the pattern remains the same as the tube is rotated about the flow direction. If a certain set of crystal planes in the left-hand side of band I is properly oriented for Bragg scattering in the red, the right-hand side must have the same planes in a different orientation which cannot Bragg scatter. Left- and right-hand are, of course, relative to observer and light source. There may be another set of planes on the right side which can Bragg scatter another colour and this is observed (often with green) but depends on the concentration and observation angle.

In region II the crystallite orientations are different from region $I$ and this results in a different set of colours for left and right side. In all the samples we have studied, region I and region II are inverted in their respective colours (i.e., region I right and region II left are the same) which implies that the crystallites in the two regions are in mirror image orientations. The regions I and II alternate spatially.

Once this pattern has been established, it remains stationary while the meniscus continues to oscillate indicating that flow is still taking place. This implies that the outer region of the colloid remains a rigid solid, while there is an inner core which is fluid as illustrated in figure 2. In fact, the large flow of the central core is easily observed by watching the motion of small inhomogeneities in the latex by transmitted light. When the oscillations are stopped, the « checkerboard " pattern remains for periods at least up to a month. This again points to the fact that the pattern originates in oriented solid regions near the surface of the tube. When inspected by transmitted light, the samples show no density fluctuations or inhomogeneities which can be associated with the periodic structure either while the flow is driven or after it has been stopped.

In order to determine how the orientations in the two regions were related to the flow direction, we performed a series of Bragg scattering studies using the $0.5145 \mu$ line of an Argon-ion laser. The « $U$ » tube was submerged in a water bath to reduce corrections from changes in index of refraction. The crystal structure of a latex of $8.2 \%$ volume fraction of $0.220 \mu$ diameter spheres was determined from its DebyeScherrer pattern to be BCC. The concentration was chosen so that no Bragg scattering would occur in a forward direction (through the tube) and so that the beam would be significantly attenuated by the time it reached the core of the $4 \mathrm{~mm}$ tube. This assured a measurement of the structure only of the solid surface regions of interest.

The latex was then driven in the manner described above until the periodic pattern was well established. The results of this study for one region are listed in table I, where they are compared to the predicted planar spacings for a FCC lattice with a particle density of $1.5 \times 10^{13} \mathrm{~cm}^{-3}$, the most densely packed plane (111) parallel to the glass surface and the (200) plane intersecting the (111) plane along a line perpen- 
Table I. - Bragg scattering data.

\begin{tabular}{|c|c|c|c|c|c|c|}
\hline \multirow[t]{2}{*}{ plane } & \multicolumn{2}{|c|}{$\begin{array}{l}d \\
(\mu \mathrm{m})\end{array}$} & \multicolumn{2}{|c|}{$\begin{array}{c}\xi \\
\angle \text { bet. normal } \\
\text { and surface }\end{array}$} & \multicolumn{2}{|c|}{$\begin{array}{c}\beta \\
\angle \text { bet. normal } \\
\text { and flow }\end{array}$} \\
\hline & expt. & th. & expt. & th. & expt. & th. \\
\hline (111) & 0.39 & 0.40 & 0 & 0 & 90 & 90 \\
\hline$(200)$ & 0.35 & 0.35 & $50-54$ & 55 & $112-116$ & 114 \\
\hline$(220)$ & 0.25 & 0.25 & $40-47$ & 35 & 125-129 & 125 \\
\hline (311) & 0.21 & 0.21 & $26-29$ & 30 & $95-100$ & 104 \\
\hline (222) & 0.22 & 0.20 & 0 & 0 & 90 & 90 \\
\hline
\end{tabular}

dicular to the flow direction. The agreement between the observed Bragg spots and their theoretical assignment is excellent. Note that the shear has transformed the crystal structure from its equilibrium BCC configuration to FCC. This is best visualized by the schematic in figure $3 a$. The hexagonal close-packed planes of the FCC crystal are in the plane of the surface of the capillary with the hexagon edges perpendicular to the flow direction. The second plane from the surface is arranged in the manner illustrated. It is immediately obvious that this orientation is the one which best resists the applied shear [14]. The easy shear direction for the FCC crystal is shown by the dashed arrow. Figure $3 b$ shows the relationship between the important crystallographic directions and the relevant axes.

When we perform the same experiments and analysis in region II (by merely displacing the tube half a period) we find that the structure is the mirror image of the structure in region I. In terms of the crystallography of the FCC lattices, region I could be labelled ABCABCABC..., while region II would then be ACBACBACB... These are the two symmetric configurations with maximum resistance to shear in the direction of flow. The fact that the steady state configuration of the solid regions should be those most resistant to shear and that either is equally probable is fairly evident. What is not so evident is why the two coexist and form periodic domains.

In order to investigate the cause of the periodicity we have performed a series of experiments to measure the wavelength (repeat distance) of the structure as a function of a number of variables. One might expect spatial periodicity to arise from a competition between volume and surface energies (as is the case for magnetic domains [15] or superconductors in the intermediate state in a magnetic field [16]), from standing elastic waves (in this case shear waves in the colloidal crystal), from instability of hydrodynamic flow in cylindrical geometry [17], from crystal nucleation in the presence of oscillatory shear, or from motion, creation, and

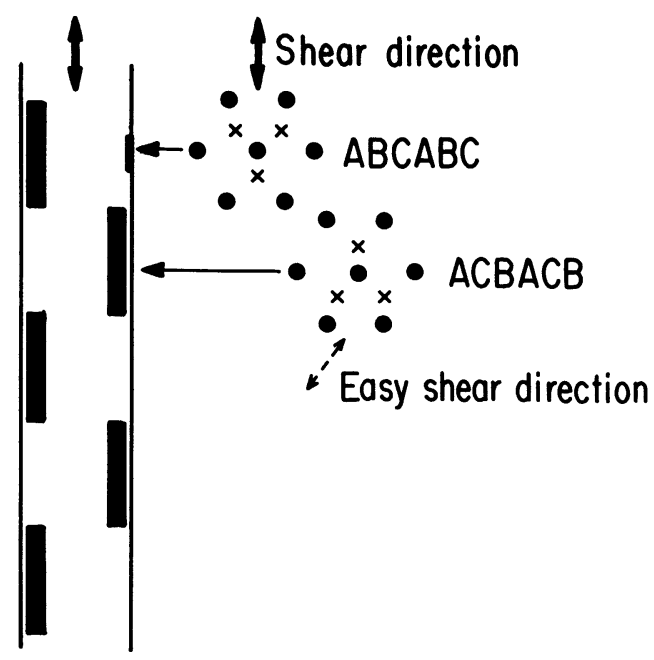

a)

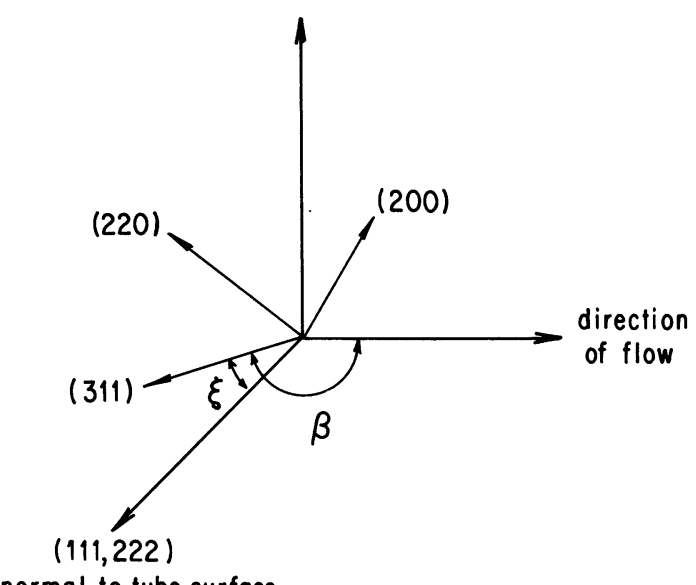

normal to tube surface

b)

Fig. 3. $-a)$ Stacking sequence in the two regions of the periodic pattern. The circles are the (111) plane against the surface of the tube. X's are the particles in the next (111) plane. b) Some crystallographic directions shown relative to the flow direction and normal to the tube surface, used with table I to determine the orientation of the crystallites as shown in $a$ ).

annihilation of driven dislocation loops. We have therefore measured the spatial periodicity as a function of amplitude of flow, frequency, shear modulus and tube diameter.

In figure 4 we have plotted the periodic wavelength as a function of amplitude of flow for samples of three different concentrations of particles and hence three different shear moduli. The shear modulus was measured for these samples in a simple mechanical apparatus described elsewhere [12]. The experiment was performed in the geometry shown in figures $1 a$ and $1 b$ by using a cathetometer to measure the periodicity and the flow amplitude. The flow amplitude (actually the flux or total flow through the central core) is measured by the peak-to-peak displacement of the meniscus. Since the diameter of the capillary is fixed and the meniscus does not appear to distort but rather 


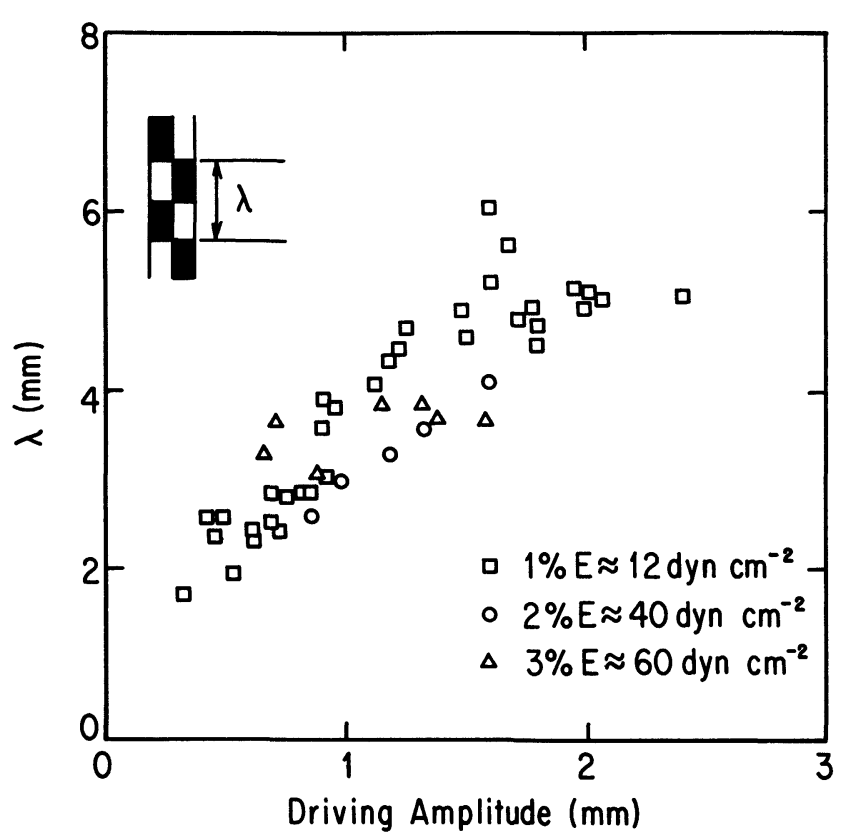

Fig. 4. - Spatial period of the striation pattern as a function of driving amplitude for three different samples with shear moduli of approximately 12, 40 and $60 \mathrm{dyn} . \mathrm{cm}^{-2}$

slides up and down the tube at these low frequencies $(2-30 \mathrm{~Hz})$, the displacement of the meniscus implies a certain volume displacement. The fluid is incompressible so the meniscus displacement is directly proportional to the total flux. The abscissa in figure 4 is the peak-to-peak displacement of the meniscus.

Three points should be noted from figure 4. 1) The wavelength increases with the amplitude of flow. As we shall see in the discussion, this argues against a simple model in which surfaces are added to reduce volume energy $[15,16]$. 2) The periodicity seems largely independent of the shear modulus. We therefore conclude that we are not observing a standing elastic wave whose wavelength should vary as the velocity (hence as the square root of the elastic constant) for fixed frequency. 3) The shear modulus provides certain limits in flow amplitude under which the periodic pattern can be seen at all.

In figure 5 we show the wavelength of the periodic structures as a function of amplitude for two different frequencies. Varying the frequency at fixed amplitude is not a well controlled experiment since the total flow and velocity in the core change as frequency changes. While at low amplitudes there does appear to be a difference between the two frequencies, at higher amplitudes the two sets of data are indistinguishable. This result also argues against a standing elastic wave.

Concentrating on the $5 \mathrm{~Hz}$ data, at low amplitude the wavelength is approximately proportional to the amplitude, while at higher values the wave length to a constant. This behaviour is seen in almost all of the samples. However, since the periodic structure only results from a certain region in amplitude-fre-

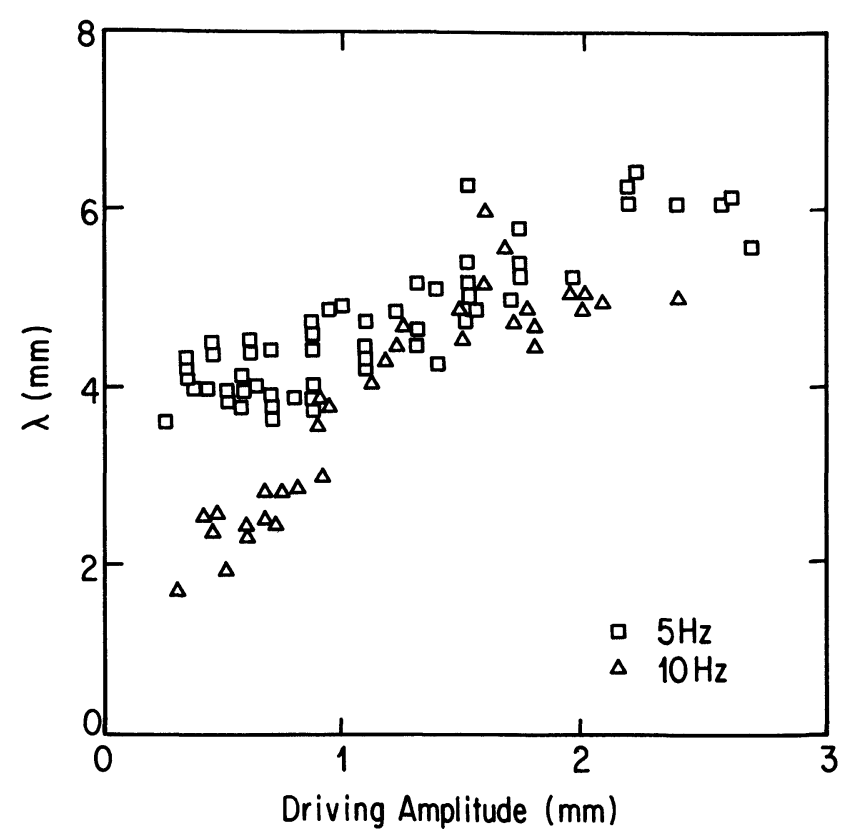

Fig. 5. - Period as a function of driving amplitude for two frequencies.

quency space for different samples, we sometimes observe only the flat region.

In many problems involving instabilities the characteristic wavelength is proportional to and the same order as a characteristic dimension of the system which in the present case would be the tube diameter. The problem in doing a study of the wavelength as a function of the tube diameter is that many of the properties of the flow change with tube diameter so that it would be misleading to plot the wavelength as a function of amplitude of displacement for different tube sizes. In such a situation the most instructive plot would be the wavelength as a function of Reynolds number which puts all of the varying quantities in dimensionless form. We take the Reynolds number as $R e=f d \rho A / \eta$ where $d$ is the diameter of the tube and $A$ is the driving amplitude, all in cgs. In figure 6 we have plotted the wavelength observed as a function of Reynolds number for several tube sizes. While no systematic variation with tube size is seen it is clear that the wavelength does not vary simply with tube diameter, and at best varies with some lower power of tube diameter such as $1 / 2$ or $1 / 3$. It is also worth pointing out that the values of Reynolds number used throughout this study are small, being one or two orders of magnitude less than required to observe turbulent behaviour for liquid flow in a tube.

Since the flow of a fluid in a tube is complicated, especially a fluid with highly nonlinear elastic properties in the region of shear rates studied, we have attempted similar experiments in a cylinder with oscillatory motion about its axis. This is shown in the photos of figure 7. At low shear rates the crystallites respond elastically (Fig. 7b). At higher shear 


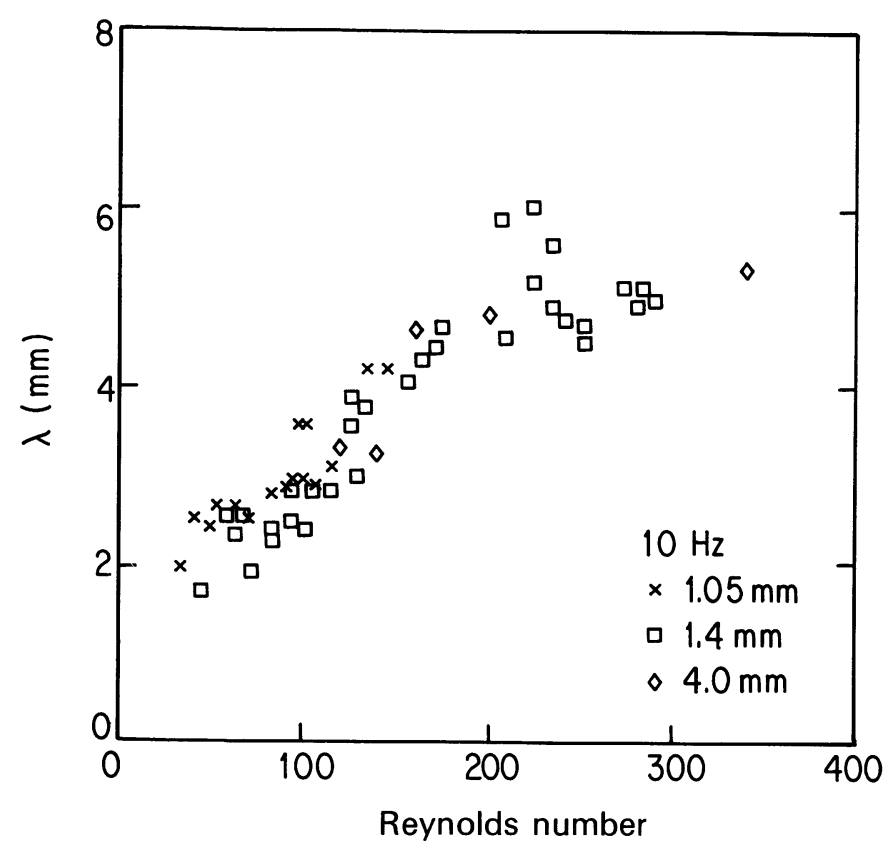

Fig. 6. - Period as a function of Reynolds number for three different tube diameters.

rates the sharp colour lines seen along the surface become uneven indicating some plastic flow. If the oscillations are allowed to continue at the same amplitude for an hour or so, a very « chaotic " pattern of large randomly oriented crystallites is observed on the cylinder surface and remains stationary (Fig. 7c). Presumably this configuration of crystallites best resists the oscillatory shear for the cylindrical geometry. It is quite reminiscent of the work hardening of metal. Although we have performed many experiments at different frequencies and amplitudes of oscillation we have never observed periodic structures in this geometry.

3. Discussion. - The nonlinear elastic properties of colloidal crystals have been studied previously by Ackerson and Clark. They have reported a transition from a $\mathrm{BCC}$ structure to a two dimensional hexagonal close-packed structure in which the planes slip past one another, and finally linear chain-like structures and a liquid state as the rms shear rate is increased $[10,18]$. Defining the time-averaged shear rate, $S$, by :

$$
S=\left\langle\left(\frac{\partial v}{\partial z}\right)^{2}\right\rangle^{1 / 2}
$$

their experiments involved values of $S$ from 4.4 to $17.9 \mathrm{~Hz}$. Although they have not measured the shear modulus of their samples $(0.109 \mu$ diameter spheres of density $\approx 10^{12} \mathrm{~cm}^{-3}$ ), they estimate a shear modulus of $E \approx 1$ dyn. $\mathrm{cm}^{-2}$. If we extrapolate our shear modulus measurement down to their densities we obtain $E \approx 0.5$ dyn. $\mathrm{cm}^{-2}$ for their samples. Their

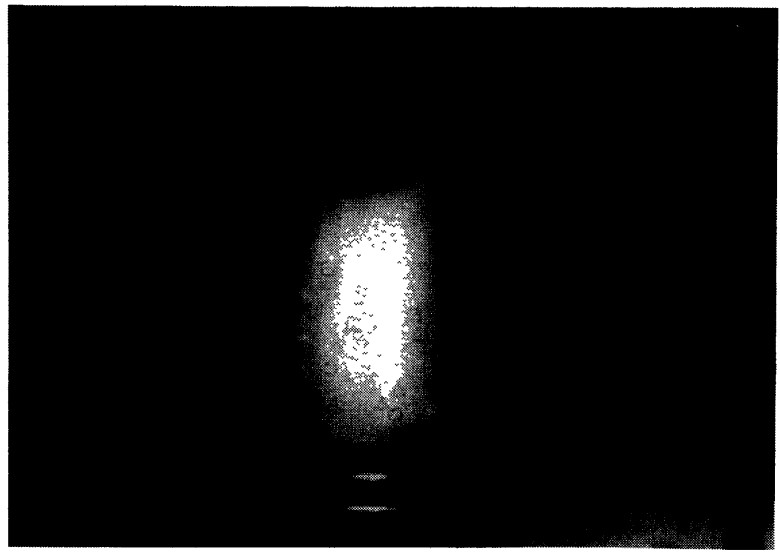

a)

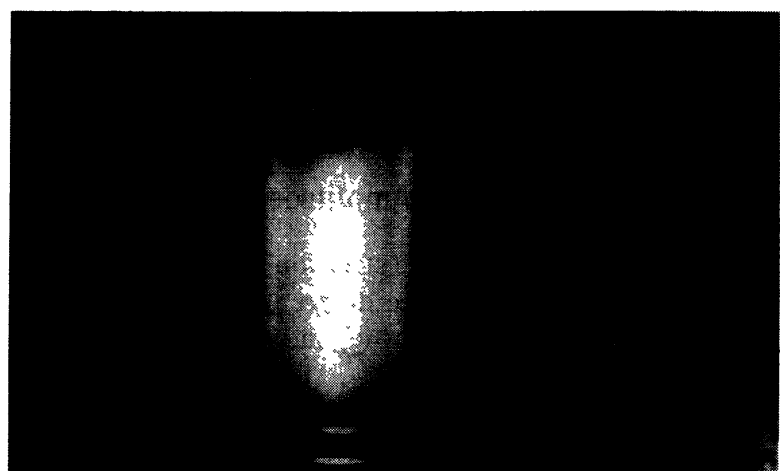

b)

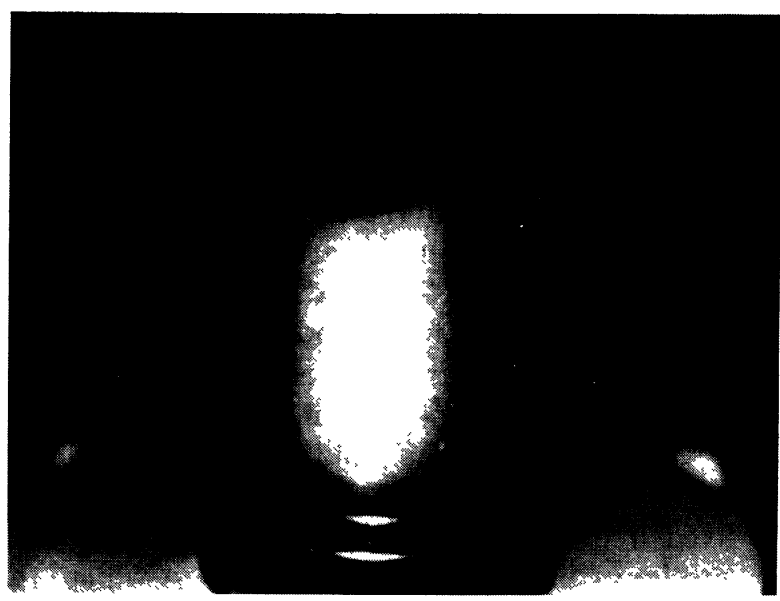

c)

Fig. 7. - Oscillatory shear about a cylindrical axis. a) Before shear. b) At low shear rate the crystallites align. c) At larger shear a static pattern of chaotically oriented crystallites results.

experiments therefore involved shear rate to shear modulus ratios in the range

$$
S / E \approx 9-36 \mathrm{~g} / \mathrm{s} .
$$

For comparison, we observe the periodic structures in samples with $E \approx 10-100$ dyn. $\mathrm{cm}^{-2}$ for shear rates $S \approx 50 \mathrm{~Hz}$. Thus the range covered by our experiments is :

$$
S / E \approx 0.5-5 \mathrm{~g} / \mathrm{s} .
$$


At higher shear rates our samples also become liquid as evidenced by the lack of opalescence. Although the comparison of shear rates should be made with the yield stress rather than the shear modulus, we assume that they are approximately proportional [5]. Thus our experiments differ from those of Ackerson et al. both in terms of geometry (they use a parallel plate cuvette) and in relative shear rate with our experiments being closer to the region where plastic deformation and flow is expected.

The first question we address is whether the configuration shown schematically in figure 2 can be stable with a fluid core and a rigid, solid region near the capillary wall. If we increase the pressure applied to a solid confined to a tube, at some pressure the solid must yield and flow. Consider a cylindrical region of the solid of radius $r$ as illustrated in figure 8. The applied force on this region is :

$$
F_{\mathrm{a}}=\pi r^{2} P \text {. }
$$

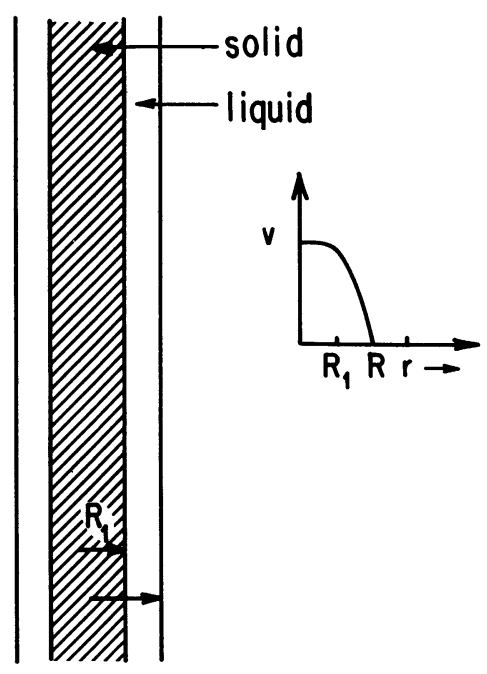

Fig. 8. - Schematic of plug flow with solid core and fluid near surface of tube. Such flow is shown to be unstable.

The restoring force on the cylinder comes from the shear stress acting on the cylindrical surface :

$$
F_{\mathrm{r}}=\pi r l \sigma
$$

where $l$ is the length of the cylinder. The inner cylindrical region will displace when $\sigma=\sigma_{\text {crit }}$, the yield stress of the solid. Equating the forces, the critical stress will be reached first for the largest allowed value of $r$ which corresponds to the radius of the tube

$$
\sigma=\frac{r P}{l}
$$

Thus the solid will yield first at the tube surface and will slide as a solid cylinder with a fluid region between it and the tube. This is the conventional situation as a cylinder yields under pressure.

The result is plug flow with a solid core that remains rigid and a liquid sheath that undergoes Poiseuille flow. The hydrodynamic equations can be solved by using the boundary conditions together with the Navier-Stokes equation. The velocity at the wall of the tube is zero and the radius of the solid core is determined by the viscous shear forces being equal to the yield stress of the solid

$v(r)=0, r=R, \quad \eta \frac{\partial v}{\partial r}=\sigma_{\text {crit }}=-\frac{\Delta P}{2 l}, \quad r=R_{1}$.

The radius of the solid is then set in terms of the pressure drop and the velocity profile and total mass flow are given by :

$$
\begin{aligned}
v(r) & =-\frac{\Delta P}{4 \eta l}\left(R^{2}-r^{2}\right), \quad R_{1}<r<R \\
v(r) & =\frac{\Delta P}{4 \eta l}\left(R^{2}-R_{1}^{2}\right), \quad r<R_{1} \\
Q & =\frac{\pi \rho \Delta P}{8 \eta l}\left(R^{4}-R_{1}^{4}\right) .
\end{aligned}
$$

The flow is stable under the assumption that the pressure drop along the tube is constant and that there is no radial pressure gradient. Unfortunately, this solution is not appropriate for the solid which cannot maintain a uniform pressure drop with no tangential pressure gradient or density gradient. Under the given conditions, the buckling of the solid rod must be considered [1].

For the problem of the buckling rod it is necessary to know Young's modulus which can be expressed in terms of the compressibility and the shear modulus. While the shear modulus and yield stress are low, the appropriate compressibility is that of the water which is quite large $\left(\approx 5 \times 10^{11}\right.$ dyn. $\mathrm{cm}^{-2}$ compared to a shear modulus of $\approx 100$ dyn. $\mathrm{cm}^{-2}$ ). Young's modulus then depends only on the shear modulus. The condition that the solid will buckle is given by :

$$
P_{\text {crit }}=\frac{3 \pi^{3} \mu R_{1}^{2}}{4 l^{2}}
$$

For our configuration and latex, buckling will occur when $P$ is $\approx 10$ dyn. $\mathrm{cm}^{-2}$, which corresponds to $\approx 10^{-5}$ bar. Our driving pressures are typically $\approx 10^{-3}$ bar when the anomalous flow pattern is seen.

Thus plug flow is unstable in this configuration with a homogeneous velocity field and experimentally we have never observed plug flow as the stable configuration.

We therefore consider the opposite case, i.e., a liquid core surrounded by a solid in contact with the capillary walls. This is shown schematically in figure 2 .

For Poiseuille flow the total mass flux is proportional to the fourth power of the radius of the tube. 
The shear is again given by the viscosity times the derivative of the velocity :

$$
\begin{aligned}
Q & =\frac{\pi \Delta P \rho}{8 \eta l} R_{2}^{4} \\
\sigma_{\text {crit }} & =\eta \frac{\partial v}{\partial r}=\frac{\Delta P}{2 l} R_{2}
\end{aligned}
$$

where $R_{2}$ is the radius of the fluid core. In order to have a stable flow configuration the tangential force must equal the yield stress and by LeChatelier's principle the tangential force must increase as the radius decreases. For fluid flow in the centre of the solid region, any decrease in the radius would increase the velocity of the fluid (at the centre) by a factor of the radius to the fourth power and would increase the shear force by the inverse third power

$$
v \propto Q / R^{4}, \quad \eta \frac{\partial v}{\partial r} \propto Q / R^{3} .
$$

Thus the radius of the fluid region is self-healing and locally stable under conditions of constant mass flow. The basic configuration of fluid inside and solid on the surface of the capillary is of course what is observed in all of our experiments.

When equation (10) is solved for the mass flow directly in terms of the pressure difference (eliminating $R_{2}$ ) the relationship is :

$$
Q=\frac{2 \pi \sigma^{4} \rho}{\eta(\Delta P / l)^{3}} .
$$

The differential impedance of this configuration is negative (flux increases as pressure decreases). Such a system cannot support a homogeneous flow along its length.

Thus the two homogeneous flow configurations which might be expected when a solid in a cylinder yields have both been shown to be unstable. The conclusion must be that an inhomogeneous flow will result with velocity and pressure gradients modulated along the length of the tube and pressure gradients non-vanishing in the radial direction as well. The situation is quite similar to that found for superconductors in the current-carrying intermediate state [16]. In this well-known case it is impossible to find a homogeneous current solution for the boundary conditions for the electric and magnetic fields in the presence of a current, the magnetic field of which exceeds the critical field at the surface of the wire. As in the present case, the cylindrical geometry is special. Although the intermediate state superconductor remains an unsolved theoretical problem, experimentally and theoretically it is known that the normal and superconducting regions form a periodic array. Unfortunately, the periodicity of the domains has never been satisfactorily calculated.
The way in which the observed configuration of liquid core and periodic regions can arise in the presence of the oscillating driving force is then as follows. Under the influence of the oscillating pressure, the yield stress is first exceeded at the wall of the tube. The result is uniform slipping of the solid cylinder with a small liquid region which separates it from the tube wall. However, once the cylinder slips the shear force is related to the velocity gradient. At the wall this force may be less than the yield stress of the solid (for particular orientations). Crystallites will then nucleate and grow inwardly from the wall constricting the flow of the central region. The constant flow condition requires that the velocity gradient increase until the shear condition in equation (7) is reached. The most stable configuration will occur when the solid crystalline region is oriented with respect to the flow in the direction with the highest yield stress. Crystallites with other orientations will erode as the velocity gradient increases due to the presence of some regions of high shear resistance.

The model derived above is particular to flow in a tube from its dependence of mass flow on radius. No such stable configuration of fluid and solid regions would result in planar geometry. Moreover, there are only certain flow conditions in which this configuration is of interest.

The pressure must be sufficiently high so that the uniform solid with random crystallite orientations is unstable as in equations (7) and (8). On the other hand the mass flow cannot be large enough to cause a liquid core which has a larger radius than the radius of the tube. In our experiments we have not measured the pressure but rather the amplitude of oscillation of the surface. This is related directly to the mass flow by :

$$
Q=\pi R^{2} \rho \omega A
$$

where $A$ is the amplitude of the oscillation. For this mass flow the average radius of the core region is given by :

$$
R_{2}=\left[\frac{4 Q \rho}{\pi \eta \sigma_{\mathrm{crit}}}\right]^{1 / 3}
$$

When the applied oscillating pressure is less than the critical value necessary to destroy the solid, the surface responds elastically. The amplitude of displacement of the surface is then related to the solid elastic constants. The maximum amplitude of displacement for which we can observe a solid near the capillary wall is when the core radius $R_{2}$ in equation (14) is equal to $R$. In fact, we observe the periodic structures only in limited regions of amplitude-frequency space, and these regions correspond roughly to the limits given above.

In conclusion we have found an instability in the driven flow of strongly interacting colloids in a capillary. The finite yield stress of the colloidal crystal can be readily exceeded and a coexistence of liquid and 
solid regions can result. Experimentally we have found a liquid core surrounded by an alternating set of well aligned crystallite regions which have formed in such a way as to best resist the shear. Theoretically we have shown that a uniform flow along the length of the tube is unstable and thus inhomogeneities must result. This is the first observation of one of the many instabilities that must result from flow in an extremely nonlinear solid/fluid which goes from finite rigidity to a low viscosity system under the influence of relatively little shear.

We would like to acknowledge many useful discussions with P. Pincus, S. Alexander, H. Hervet, T. Holstein and P. Pieranski.

\section{References}

[1] VAN Den Hul, H. J. and VAnderhof, J. W., J. Colloid Interface Sci. 28 (1968) 336.

[2] Hiltner, P. A. and Krieger, I. M., J. Phys. Chem. 73 (1969) 2386.

[3] Crandall, R. S., Williams, R. and Wojitowicz, P. J., Phys. Rev. Lett. 37 (1976) 348.

[4] Schaeffer, D. W. and Ackerson, B. J., Phys. Rev. Lett. 35 (1975) 1448.

[5] Mitaku, S., Ohtsuki, T., Enari, T., Kishimoto, A. and Okano, K., Japan. J. Appl. Phys. 17 (1978) 305.

[6] Homola, A. and Robertson, A. A., J. Colloid Interface Sci. 54 (1976) 286.

[7] Clark, N. A., Hurd, A. J. and Ackerson, B. J., Nature 57 (1979) 281.

[8] Pieranski, P., Les Houches Summer School, 1981, to be published.

[9] Crandall, R. S. and Williams, R., Science 198 (1977) 293.
[10] Clark, N. A. and Ackerson, B. J., Phys. Rev. Lett. 44 (1980) 1005 ;

Ackerson, B. J. and Clark, N. A., Phys. Rev. Lett. 46 (1981) 123.

[11] Pieranski, P., Dubois-Violette, E., Rothen, F. and STrZelecki, L., J. Physique 41 (1980) 369.

[12] Lindsay, H. M. and Chaikin, P. M., Bulletin A.P.S. 26 (1981) 306 and to be published.

[13] Rexyn I-300 (H-OH) research grade, Fisher Scientific Co., Fair Lawn, N. J.

[14] Friedel, J., Dislocations (Pergamon Press, Oxford) 1964.

[15] KITTEL, C., Introduction to Solid State Physics (J. Wiley and Sons, New York) 1976.

[16] London, F., Superfluids, Vol. I (Dover Publications, New York) 1961.

[17] Landau, L. D. and Lifshitz, E. M., Fluid Mechanics (Pergamon Press, Oxford) 1978.

[18] Landau, L. D. and Lifshitz, E. M., Elasticity (Pergamon Press, Oxford) 1978. 